\title{
SOLUTIONS OF THE EINSTEIN EQUATIONS INVOLVING FUNCTIONS OF ONLY ONE VARIABLE*
}

\author{
BY
}

EDWARD KASNER

Taking the element of length in the orthogonal form

$$
d s^{2}=\lambda_{1} d x_{1}^{2}+\lambda_{2} d x_{2}^{2}+\lambda_{3} d x_{3}^{2}+\lambda_{4} d x_{4}^{2}
$$

we assume that the potentials $\lambda$ are functions of a single variable $x_{1}$. We shall find the forms of these functions for which the Einstein cosmological equations

$$
R_{i k}-\frac{1}{4} R g_{i k}=0
$$

are satisfied. The results are given in (14), (20), and (17) below. They involve only elementary functions, and the corresponding four-dimensional manifolds can be represented as imbedded in flat space of seven dimensions.

Making use of the formulas for $R_{i k}$ calculated in a previous papert we find that, of the ten equations (2), the six with unlike subscript vanish identically, while the four with like subscripts become

(3)

$$
\begin{aligned}
L_{211}+L_{311}+L_{411}+L_{21}^{2}+ & L_{31}^{2}+L_{41}^{2}-L_{21} L_{31}-L_{31} L_{41} \\
& -L_{41} L_{21}-L_{11}\left(L_{21}+L_{31}+L_{41}\right)=0 \\
L_{211}-L_{311}-L_{411}+L_{21}^{2}- & L_{31}^{2}-L_{41}^{2}+L_{21} L_{31}-L_{31} L_{41} \\
& +L_{41} L_{21}-L_{11}\left(L_{21}-L_{31}-L_{41}\right)=0 \\
-L_{211}+L_{311}-L_{411}-L_{21}^{2}+ & L_{31}^{2}-L_{41}^{2}-L_{21} L_{31}+L_{31} L_{41} \\
- & L_{41} L_{21}-L_{11}\left(-L_{21}+L_{31}-L_{41}\right)=0 \\
-L_{211}+L_{311}+L_{411}-L_{21}^{2}- & L_{31}^{2}+L_{41}^{2}-L_{21} L_{31}-L_{31} L_{41} \\
& +L_{41} L_{21}-L_{11}\left(-L_{21}-L_{31}+L_{41}\right)=0 .
\end{aligned}
$$

* Presented to the Society, September 7, 1921.

† Kasner, The solar gravitational field completely determined by its light rays, Mathematische Annalen, vol. 85 (1922), p. 227. 
Here $L_{i}=\frac{1}{2} \log \lambda_{i}$ and the second and third subscripts indicate differentiation with respect to $x_{1}$, while the first subscript states merely the name of the function.

The four equations (3) are not independent, their sum being identically zero. They thus reduce to three equations. We may assume without loss of generality that $\lambda_{1}=1$, for by applying the transformation

$$
\lambda_{1} d x_{1}^{2}=d x_{1}^{* 2}
$$

to the form (1), it reduces to

$$
d s^{2}=d x_{1}^{* 2}+\lambda_{2}^{*} d x_{2}^{2}+\lambda_{3}^{*} d x_{3}^{2}+i_{4}^{*} d x_{4}^{2},
$$

where the $\lambda_{i}^{*}$ are three new functions of the new variable $x_{1}^{*}$. Thus the functions $L_{i}$ reduce to

$$
L_{1}^{*}=0, \quad L_{i}^{*}=\frac{1}{2} \log \hat{\lambda}_{i}^{*} \quad(i=2,3,4) .
$$

We then obtain from equations (3) by adding the first and second, first and third, and the first and fourth, respectively

$$
\begin{aligned}
& L_{211}^{*}+L_{21}^{*}-L_{31}^{*} L_{41}^{*}=0, \\
& L_{311}^{*}+L_{31}^{*}-L_{41}^{*} L_{21}^{*}=0, \\
& L_{411}^{*}+L_{41}^{*}-L_{21}^{*} L_{31}^{*}=0 .
\end{aligned}
$$

We now introduce three new functions

$$
x=L_{21}^{*}, \quad y=L_{31}^{*}, \quad z=L_{41}^{*},
$$

and denote the independent variable $x_{1}^{*}$ by $t$, thus reducing our system (5) to the simple form

$$
\begin{aligned}
& x^{\prime}=y z-x^{2}, \\
& y^{\prime}=z x-y^{2}, \\
& z^{\prime}=x y-z^{2},
\end{aligned}
$$

a system of three ordinary differential equations, primes denoting differentiation with respect to $t$. 
SOLUTION OF THE SYSTEM (6)

In order to solve the system (6) we first reduce it by means of the substitution

$$
\xi=x+y+z, \quad \eta=x y+y z+z x, \quad \zeta=x y z
$$

to the more easily integrable form

$$
\begin{aligned}
\xi^{\prime}+\xi^{2} & =3 \eta, \\
\eta^{\prime} & =0, \\
\zeta^{\prime}+3 \xi \zeta & =\eta^{2} .
\end{aligned}
$$

The second of these equations shows that $\eta$ is constant and we write for later convenience

$$
\eta=-\frac{c^{2}}{3}
$$

Then the first becomes

$$
\xi^{\prime}+\xi^{2}+c^{2}=0
$$

the solution of which (unless $\xi \xi$ is constant, which special case is treated later)

$$
\xi=c \tan c(k-t)
$$

where $k$ is an arbitrary constant of integration. (The special case where $c=0$, that is, $\eta=0$, is treated separately later.)

By $\left(6^{\prime}\right)$ the three functions $x, y, z$ are the roots of the cubic equation

$$
x^{3}-\xi x^{2}+\eta x-\zeta=0
$$

Substituting

$$
x=X+\frac{1}{3} \xi
$$

and using (8) and (9), this reduces to

$$
X^{3}-\frac{1}{3} c^{2} \sec ^{2} c(k-t) \cdot X-\left(\zeta+\frac{2}{27} \xi^{3}-\frac{1}{3} \xi \eta\right)=0 .
$$

We observe that the third equation in (7) is linear in $\zeta$ and has for a particular solution*

$$
\zeta=-\frac{2}{27} \xi^{3}+\frac{1}{3} \xi \eta
$$

* This was suggested to me by Dr. Gronwall, whom I wish to thank, as well as Dr. Reddick, for simplifications in my original calculations. 
and hence we obtain its general solution in the form

$$
\zeta=-\frac{2}{27} \xi^{3}+\frac{1}{3} \xi \eta+c_{1} c^{3} \sec ^{3} c(k-t),
$$

where $c_{1}$ is an arbitrary constant of integration. If now we divide the roots of the cubic (10) by $c \sec c(k-t)$, that is, employ the substitution

$$
X=a c \sec c(k-t)
$$

it becomes

$$
a^{3}-\frac{1}{3} a-c_{1}=0
$$

so that the new unknown $a$ is actually constant and dependent on $c_{1}$. We then have as a general solution of equations (6)

$$
x, y, z=\frac{1}{3} c \tan c(k-t)+a_{i} c \sec c(k-t),
$$

where $a_{i}$ takes on successively the values which are the roots of the cubic (12). The result thus involves three arbitrary constants $c, k, c_{1}$.

The PRINGIPAL QUADRATIC FORM IN ONE VARIABLE

Going back now to the original notation by means of $\left(5^{\prime}\right)$ and integrating the expressions for $x, y, z$ given in (13), we obtain

$L_{i}^{*}=\frac{1}{2} \log \lambda_{i}^{*}=\frac{1}{3} \log \cos \left(k-x_{1}^{*}\right)+a_{i} \log \left\{\sec c\left(k-x_{1}^{*}\right)+\tan c\left(k-x_{1}^{*}\right)\right\}$.

Therefore

$$
\lambda_{i}^{*}=\cos ^{2 / 3} c\left(k-x_{1}^{*}\right)\left\{\sec c\left(k-x_{1}^{*}\right)+\tan c\left(k-x_{1}^{*}\right)\right\}^{2 a_{i}} \quad(i=2,3,4)
$$

are the general values of the coefficients in the differential form (4).

We now return to the general orthogonal form (1) by the transformation

$$
d x_{1}^{* 2}=\frac{4 d x_{1}^{2}}{c^{2}\left(1+x_{1}^{2}\right)}
$$

that is,

$$
\cos c\left(k-x_{1}^{*}\right)=\frac{2 x_{1}}{1+x_{1}^{2}}
$$


which eliminates all the trigonometric functions, giving

$$
\lambda_{1}=\frac{4}{c^{2}\left(1+x_{1}^{2}\right)^{2}}, \quad \lambda_{i}=\left(\frac{2 x_{1}}{1+x_{1}^{2}}\right)^{2 / 3} x_{1}^{2 a_{i}} \quad(i=2,3,4) .
$$

We have thus established our main theorem:

The principal solution, in orthogonal form (1) where the coefficients are functions of $x_{1}$ alone, of Einstein's cosmological equations

$$
R_{i k}-\frac{1}{4} g_{i k} R=0,
$$

is given by

$$
d s^{2}=\frac{4 d x_{1}^{2}}{c^{2}\left(1+x_{1}^{2}\right)^{2}}+\left(\frac{2 x_{1}}{1+x_{1}^{2}}\right)^{2 / 3}\left\{x_{1}^{2 a_{2}} d x_{2}^{2}+x_{1}^{2 a_{3}} d x_{3}^{2}+x_{1}^{2 a_{4}} d x_{4}^{2}\right\}
$$

where $c$ is an arbitrary constant and $a_{2}, a_{3}, a_{4}$ are the roots of a cubic of type (12), that is, are constants obeying the relations

$$
\begin{aligned}
a_{2}+a_{3}+a_{4} & =0 \\
a_{2} a_{3}+a_{3} a_{4}+a_{4} a_{2} & =-\frac{1}{3} .
\end{aligned}
$$

The potentials in this form are in general intercendental (transcendental) functions, since the exponents are usually irrational. A simple example where the exponents are rational numbers and hence the potentials $g_{i k}$ are algebraic functions is given by

$$
a_{2}=\frac{1}{3}, \quad a_{3}=\frac{1}{3}, \quad a_{4}=-\frac{2}{3} .
$$

(This of course does not mean that the finite four-dimensional spread is necessarily algebraic.)

SPECIAL CASE WHERE THE CONSTANT $c$ VANISHES

In this special case $\eta=0$, so that from (7) we find

$$
\xi=\frac{1}{t-t_{0}} \text { or } \xi=0
$$

For the first form we find $\zeta=c_{2} /\left(t-t_{0}\right)^{3}$, and finally,

$$
\begin{aligned}
x= & \underset{t-t_{0}}{\lambda}, \quad y=\frac{\mu}{t-t_{0}}, \quad z=\frac{\nu}{t-t_{0}}, \\
\lambda+\mu+\nu=1, \quad \lambda \mu+\mu \nu+\nu \lambda & =0 .
\end{aligned}
$$


For the second form, $\zeta=$ constant $=c_{3}^{3}$, and

$$
x=c_{3}, \quad y=\omega c_{3}, \quad z=\omega^{2} c_{3},
$$

where $\omega$ is an imaginary cube root of unity.

Thus the quadratic form is either

or else

$$
\begin{aligned}
& d s^{2}=d x_{1}^{2}+x_{1}^{2 \lambda} d x_{2}^{2}+x_{1}^{2 \mu} d x_{3}^{2}+x_{1}^{2 \nu} d x_{4}^{2}, \\
& d s^{2}=d x_{1}^{2}+e^{2 c_{3} x_{1}} d x_{2}^{2}+e^{2 \omega c_{3} x_{1}} d x_{3}^{2}+e^{2 \omega^{2} c_{3} x_{1}} d x_{4}^{2} .
\end{aligned}
$$

Both of these solutions may be included by transformation in the single form*

$$
\begin{gathered}
d s^{2}=x_{1}^{2 a_{1}} d x_{1}^{2}+x_{1}^{2 a_{2}} d x_{2}^{2}+x_{1}^{2 a_{3}} d x_{3}^{2}+x_{1}^{2 a_{4}} d x_{4}^{2}, \\
a_{2}+a_{3}+a_{4}=a_{1}+1, \quad a_{2}^{2}+a_{3}^{2}+a_{4}^{2}=\left(a_{1}+1\right)^{2} .
\end{gathered}
$$

The simplest solution in integers of the two conditions on the exponents in (17) is $a_{1}=2, a_{2}=2, a_{3}=2, a_{4}=-1$, giving

$$
d s^{2}=x_{1}^{4}\left(d x_{1}^{2}+d x_{2}^{2}+d x_{3}^{2}\right)+x_{1}^{-2} d x_{4}^{2} .
$$

By a misprint in the American Journal of Mathematics, the last term is incorrectly written $x_{4}^{-2} d x_{4}^{2}$.

SPECIAL CASE WHERE THE FUnCtion $\xi$ is CONSTANT

Going back to $\left(8^{\prime}\right)$, it was noted that the solution (9) does not include the case where $\xi$ is constant. In that case, replacing $i c$ by $k$, we have from (7)

$$
\xi=k, \quad \eta=\frac{k^{2}}{3}, \quad \zeta=\frac{k^{3}}{27}+C_{1} e^{-3 k t},
$$

where $C_{1}$ is a new constant of integration. We then find

$$
\begin{aligned}
& x=\frac{k}{3}+C_{1} e^{-l t}, \\
& y=\frac{k}{3}+\omega C_{1} e^{-k t}, \\
& z=\frac{k}{3}+\omega^{2} C_{1} e^{-l l t},
\end{aligned}
$$

* This result is the solution of the Einstein field equations in their first form, $R_{i k}=0$, and was first given by the writer in a paper read before the Society in September, 1920 (see Bulletin of the American Mathematical Society, vol. 27 (1920-21), p. 62). See also the American Journal of Mathematics, vol. 43 (1921), p. 220. The same problem was later treated by Murnaghan and Eisenhart. The general results of the present paper were given in Science, vol. 54 (1921), pp. 304-305. 
where $\omega$ is, as above, an imaginary cube root of unity. From these values, using $\left(5^{\prime}\right)$, we find the three functions $L_{i}^{*}$; and finally by a simple transformation and a change of constants the final form is obtained

$$
d s^{2}=\frac{d x_{1}^{2}}{k^{2} x_{1}^{2}}+x_{1}^{2 / 3}\left\{e^{C x_{1}^{-1}} d x_{2}^{2}+e^{\omega C x_{1}^{-1}} d x_{3}^{2}+e^{\omega^{2} C x_{1}^{-1}} d x_{4}^{2}\right\} .
$$

\section{SUMMARY OF SOLUTIONS}

For the symmetric system of differential equations (6) all solutions are included in (13), (19), (15) and (16). We observe that (13) involves three arbitrary constants, (19) and (15) involve two, and (16) involves only one.

For our original problem, all solutions of the cosmological equations are included in (14), (20), and (17).

We note that the results involve only elementary functions. In general the potentials, that is the coefficients $\lambda_{i}$ in the expression $d s^{2}$, are transcendental, but for an infinitude of special values of the constants involved they reduce to algebraic functions.

A form which is equivalent to the main solution (14) is

$$
\begin{aligned}
d s^{2} & =d x_{1}^{2}+\lambda_{2} d x_{2}^{2}+\lambda_{3} d x_{3}^{2}+\lambda_{4} d x_{4}^{2} \\
\lambda_{i} & =\left(\frac{1-T^{2}}{1+T^{2}}\right)\left(\frac{1-T}{1+T}\right)^{2 \alpha_{i}}, \quad T=\tan \frac{c x_{1}}{2},
\end{aligned}
$$

where $\alpha_{2}, \alpha_{3}, \alpha_{4}$ are the roots of a cubic equation of the form

$$
\alpha^{3}-\frac{1}{3} \alpha-c_{1}=0
$$

the constant $c_{1}$ being arbitrary as well as $c$.

\section{REPRESENTATION IN SPACE OF SEVEN DIMENSIONS}

We now prove the following result:

All the solutions obtained may be represented in finite form by manifolds of four dimensions imbedded in a flat space of seven dimensions, the finite equations involving integrals of elementary functions.

We first observe that all quadratic forms of the type

$$
d s^{2}=\lambda_{1}\left(x_{1}\right) d x_{1}^{2}+\lambda_{2}\left(x_{1}\right) d x_{2}^{2}+\lambda_{3}\left(x_{1}\right) d x_{3}^{2}+\lambda_{1}\left(x_{1}\right) d x_{4}^{2}
$$

are of class three, that is, are reducible to the sum of seven squares of exact differentials. 
We may write (21) as follows:

$$
d s^{2}=d s_{2}^{2}+d s_{3}^{2}+d s_{4}^{2}=Q_{2}+Q_{3}+Q_{4},
$$

where

$$
d s_{i}^{2}=Q_{i}=\frac{1}{3} \lambda_{1}\left(x_{1}\right) d x_{1}^{2}+\lambda_{i}\left(x_{1}\right) d x_{i}^{2} \quad(i=2,3,4)
$$

Here $Q_{i}$ is a quadratic form in only two variables, $x_{1}$ and $x_{i}$, and is the fundamental form of a surface of revolution. Thus our element (21) is the sum of the elements of three surfaces of revolution.

Since the coördinate $x_{1}$ is common to the three forms $Q_{i}$ we may take the three surfaces about a common axis of revolution. Denote this by $X_{1}$, and construct the first surface in a three-flat $X_{1} X_{2} X_{3}$, the second surface in a three-flat $X_{1} X_{4} X_{5}$, the third surface in a three-flat $X_{1} X_{6} X_{7}$. Here $X_{1}, X_{2}, \cdots, X_{7}$ are cartesian coördinates in a flat space of seven dimensions. The three surfaces of rotation thus have a common axis but are contained in three distinct three-flats which are mutually orthogonal.

The general theory of what I have called separable or summable* quadratic differential forms in any number of variables is a subject of considerable interest. The problem is to represent the given form in $n$ variables as the sum of two or more forms each involving fewer than $n$ variables. We must distinguish the case where the variables used in the summands are completely independent, and the case where dependent variables are introduced. The problem discussed in the present paper comes under the latter case (since the three binary forms in $\left(21^{\prime}\right)$ do not involve completely independent variables), while the problem of an earlier paper in these Transactionst gives an example of complete separability.

* Kasner, Separable quadratic differential forms and Einstein solutions, Proceedings of the National Academy of Sciences, vol.11 (1925), pp.95-96, and abstracts in the Bulletin of the American Mathematical Society.

$\dagger$ An algebraic solution of the Einstein equations, vol. 27 (1925), pp. 101-105.

Columbia University,

New YoRK, N. Y. 